PAPER

\title{
Firing Patterns in Coupled Systems of Inhibitory Neurons
}

\author{
Kaiji Kato and Hiroyuki Kitajima \\ Faculty of Engineering, Kagawa University \\ 2217-20 Hayashi, Takamatsu, Kagawa 761-0396, Japan \\ E-mail: s15g458@stu.kagawa-u.ac.jp,kitaji@eng.kagawa-u.ac.jp
}

\begin{abstract}
We investigate synchronization in neuronal networks with a small-world-like structure. Inhibitory connected neurons are considered. In a previous study, we found an interesting quasi-periodic solution such that neurons are classified into three groups: two groups with distinct firing rates and one group with only subthreshold oscillations. In this paper, we study its generation mechanism in the smallest system in which we can reconnect one synapse. As a result, we obtain the following mechanism: (1) non-uniformity of the firing frequency appears as a result of asymmetrical coupling, (2) neurons with a higher firing frequency suppress neurons with a lower firing frequency for an appropriate coupling coefficient, (3) three clustered states, that is, neurons with firing frequencies $A$ and $B(A / B$ is irrational) and neurons that do not fire, are generated. We also confirm this mechanism in a large population.
\end{abstract}

Keywords: bifurcation, small-world network, quasi-periodic solution

\section{Introduction}

Recently, complex network structures, such as smallworld [1] and scale-free [2,3] structures, have been found in various real neuronal networks $[4,5]$. Synchronization in neuronal networks has also been found and synchronous activities play an important role in information processing in the brain [6]. On the other hand, they may cause several neurological diseases such as epilepsy and tremor in Parkinson's disease [7]. Thus, the studies of synchronization in complex networks are very important and have attracted much interest. Barahona and Pecora developed master stability function (MSF) analysis to study synchronizability in complex networks [8], and Nishikawa and Motter extended it for an asymmetric case [9]. Since the average path length becomes short in small-world networks, synchronization is achieved more easily than in a regular lattice [10-13]. However, this is not the only condition; synchronizability also depends on the network size, the degree distribution (distribution of the number of links), the clustering coefficient, and so forth [14-17]. These studies are mainly based on the idea that the connections between cells promote in-phase synchronization. Thus, short-cuts in small-world networks can enhance synchronizability. However, in general it is considered that an inhibitory connection generates antiphase synchronization. Although inhibitory networks in the brain are important to generate gamma rhythms
[18-27], further studies of such inhibitory networks are required.

In our previous study [28], we observed an interesting solution in small-world networks composed of only inhibitory neurons, which have three clustered groups: firing with frequencies $\mathrm{A}$ and $\mathrm{B}$ (A/B is irrational) and subthreshold oscillations. In the full dimension, this solution is quasi-periodic; however, the time series of the oscillatory solution with firing frequency $\mathrm{A}$ or B appears to be almost periodic.

In this paper, we investigate the generation mechanism of this solution using the simplest system in which we can reconnect synapses from the second-nearest-neighbor coupling. We consider two systems: the coupled MorrisLecar (ML) and Bonhöffer-van der Pol (BVP) neurons. In a regular network, we observe a synchronous periodic solution. Upon increasing the value of the reconnecting synaptic conductance, the periodic solution undergoes subcritical period-doubling bifurcation and a stable quasi-periodic solution appears as an attractor. This synapse connects the neurons with distinct firing frequencies. Upon further increasing the value of the conductance, the firing of the neuron with a lower firing frequency disappears. Then, we observe the three clustered states. We also confirm a similar mechanism in a system with a large number of neurons. 


\section{Model Equations}

We consider networks composed of ML or BVP neurons with inhibitory synapses.

\subsection{ML model}

The ML neurons [29] with synaptic coupling are described by

$$
\begin{aligned}
C \frac{d V_{i}}{d t}= & -g_{L}\left(V_{i}-V_{L}\right)-g_{C a} M_{\infty_{i}}\left(V_{i}-V_{C a}\right) \\
& -g_{K} N_{i}\left(V_{i}-V_{K}\right)+I_{e x t}+I_{s y n_{i}} \\
\frac{d N_{i}}{d t}= & \frac{N_{\infty_{i}}-N_{i}}{\tau_{N_{i}}} \\
\frac{d s_{i}}{d t}= & \frac{1-s_{i}}{1+\exp \left(-V_{i}\right)}\left(\frac{1}{\tau_{r}}-\frac{1}{\tau_{d}}\right)-\frac{s_{i}}{\tau_{d}} \\
M_{\infty_{i}}= & 0.5\left[1+\tanh \left\{\left(V_{i}-V_{a}\right) / V_{b}\right\}\right] \\
N_{\infty_{i}}= & 0.5\left[1+\tanh \left\{\left(V_{i}-V_{c}\right) / V_{d}\right\}\right] \\
\tau_{N_{i}}= & 1 /\left[\phi \cosh \left\{\left(V_{i}-V_{c}\right) /\left(2 V_{d}\right)\right\}\right]
\end{aligned}
$$

where $V_{i}[\mathrm{mV}], N_{i}$, and $s_{i}$ are the membrane potential, the activation variable for $\mathrm{K}^{+}$, and the gating variable for the synapse, respectively. $\tau_{r}$ and $\tau_{d}$ [ms] are the rise and decay times of the synapse, respectively. $I_{\text {ext }}\left[\mu \mathrm{A} / \mathrm{cm}^{2}\right]$ is the external DC current. $I_{\text {syn }_{i}}$ is the synaptic current given by

$$
I_{\text {syn }_{i}}=\left(V_{\text {syn }}-V_{i}\right) \sum_{j=1}^{n} G_{s y n_{i j}} w_{i j} s_{j}
$$

where $G_{\text {syn }_{i j}}\left[\mathrm{mS} / \mathrm{cm}^{2}\right]$ is the maximum synaptic conductance between the $i$ th and $j$ th neurons, $w_{i j}$ is a coupling coefficient $\left(w_{i j}=1\right.$ if the $i$ th and $j$ th neurons are connected, $w_{i j}=0$ otherwise), and $V_{\text {syn }}$ is the reversal potential. Here, we consider bidirectional coupling, thus $G_{s y n_{i j}}=G_{s y n_{j i}}$ and $w_{i j}=w_{j i}$. We define the threshold value for firing as $V_{i}=0$. The values of $\left(\tau_{r}, \tau_{d}, V_{\text {syn }}\right)$ are fixed as $(0.5,7.0,-60.0)$ for the inhibitory synapse [30].

\subsection{BVP model}

The BVP equations [31] with synaptic coupling are given by

$$
\begin{aligned}
& \frac{d x_{i}}{d t}= c\left(x_{i}-\frac{1}{3} x_{i}^{3}+y_{i}+z_{i}\right) \\
& \frac{d y_{i}}{d t}=-\frac{1}{c}\left(x_{i}+b y_{i}-a\right) \\
& \frac{d s_{i}}{d t}= \frac{1-s_{i}}{1+\exp \left(-10 x_{i}\right)}\left(\frac{1}{\tau_{r}}-\frac{1}{\tau_{d}}\right)-\frac{s_{i}}{\tau_{d}} \\
&(i=1, \cdots, n)
\end{aligned}
$$

Table 1 Fixed values of system parameters for ML neuron

\begin{tabular}{l|c||c|c}
\multicolumn{1}{l|}{ ron } \\
parameter & value & parameter & value \\
\hline \hline$g_{C a}$ & $4.0\left[\mathrm{mS} / \mathrm{cm}^{2}\right]$ & $V_{a}$ & $-1.2[\mathrm{mV}]$ \\
\hline$g_{K}$ & $8.0\left[\mathrm{mS} / \mathrm{cm}^{2}\right]$ & $V_{b}$ & $18[\mathrm{mV}]$ \\
\hline$g_{L}$ & $2.0\left[\mathrm{mS} / \mathrm{cm}^{2}\right]$ & $V_{c}$ & $2[\mathrm{mV}]$ \\
\hline$V_{C a}$ & $120[\mathrm{mV}]$ & $V_{d}$ & $17.4[\mathrm{mV}]$ \\
\hline$V_{K}$ & $-80[\mathrm{mV}]$ & $C$ & $20\left[\mu \mathrm{F} / \mathrm{cm}^{2}\right]$ \\
\hline$V_{L}$ & $-60[\mathrm{mV}]$ & $\phi$ & $1 / 15\left[\mathrm{~s}^{-1}\right]$ \\
\hline \hline
\end{tabular}

$z_{i}$ models the synaptic current and is given by

$$
z_{i}=\left(Z-x_{i}\right) \sum_{j=1}^{n} g_{i j} w_{i j} s_{j}
$$

where $Z, g_{i j}$, and $w_{i j}$ are the reversal potential, the maximum coupling conductance, and the coupling coefficient, respectively.

The values of $\left(\tau_{r}, \tau_{d}, Z, g_{i j}\right)$ are fixed as $(0.09,1.2$, $-1.0,0.1)$ for modeling the inhibitory synapse. We fix the values of the parameters as $(a, b, c)=(-0.4,0.6,1.5)$ for the occurrence of an oscillation [32].

\section{Results}

We consider the simplest model in which we can reconnect synapses from the second-nearest-neighbor coupling (regular network). Thus, the number of neurons is six and the network structure is shown in Fig. 1(b), which is obtained from the regular network in Fig. 1(a) by cutting the connection between 1 and 2 and reconnecting 1 and 4 . Note that if we cut the connection between 1 and 3 and reconnect 1 and 4, then the system equations are the same owing to symmetry. The results for ML and BVP neurons with the network structure shown in Fig. 1(b) are given in Sects. 3.1 and 3.2, respectively. We show the results for a large population in Sect. 3.3.

\subsection{ML model}

We investigate synchronous firing and phase synchronization in coupled ML neurons with inhibitory synapses. The control parameter is $G_{s y n_{14}}$ and the values of other $G_{s y n_{i j}}$ are fixed as 3. Other parameter values are fixed as shown in Table 1 [33].

Figure 2 shows a one-parameter bifurcation diagram changing the value of the reconnected synaptic conductance. The value of the external DC current is fixed as 78.55 to generate tonic spiking with a frequency of 20 [Hz]. The values of $V_{5}$ on the cross section defined by

$$
\Sigma=\left\{X \in R^{3 n-1} \mid V_{1}=0, d V_{1} / d t>0\right\}
$$


are plotted. From $G_{s y n_{14}}=0.0$ to 1.15 , the system has a periodic solution. At $G_{s y n_{14}}=1.15$ subcritical perioddoubling bifurcation occurs and the stable synchronized solution becomes unstable. Then, a quasi-periodic solution appears as an attractor. We confirm that the largest Lyapunov exponent is almost zero. This quasi-periodic solution exists for $G_{s y n_{14}}>1.15$. Note that Fig. 2 shows the values of $V_{5}$ on the section defined by Eq. (5), in which the value of $V_{1}$ is constant. From this figure, we can see that $V_{1}$ and $V_{5}$ are almost synchronized for $G_{\text {syn }_{14}}>2.7$.

Figure 3 shows average firing rates (frequencies) in the parameter range between $G_{s y n_{14}}=1.0$ and 3.0. Before the bifurcation $\left(G_{s_{s n_{14}}}<1.15\right)$, a periodic solution exists; thus, the firing rate of $V_{1}\left(=V_{2}\right)$ divided by that of $V_{4}\left(=V_{5}\right)$ is a rational number (in Fig. 3, it is 2). After the bifurcation $\left(G_{s n_{14}}>1.15\right)$, the firing rate of neuron 4 is decreased rapidly. Neuron 1 has a higher firing rate than neuron 4. Thus, increasing the value of $G_{s y n_{14}}$ (coupling strength between neurons 1 and 4) causes neuron 1 to inhibit the membrane potential of neuron 4 . As a result, the firing of neuron 4 disappears at $G_{s y n_{14}} \simeq 2.7$. When $G_{\text {syn }_{14}}>2.7$, the firing rates of neurons 1 and 5 are almost the same and $V_{2}$ shows tonic spiking with a frequency of 20 [Hz] which means that neuron 2 is isolated from the network (Recall that the single neuron has a firing frequency of $20[\mathrm{~Hz}]$.). In this parameter region, it seems that neurons 1,2 , and 5 produce almost periodic spikes.

To examine the periodicity, we calculate the coefficient of variation $\left(C_{v}\right)$ of the interspike interval (ISI), which is defined by the standard deviation divided by the mean of the ISI distribution. If we obtain the ISI distribution $p(t)$, then the mean and standard deviation are given by

$$
\bar{t}=\int_{0}^{\infty} t p(t) d t, \sigma_{t}=\sqrt{\int_{0}^{\infty}(t-\bar{t})^{2} p(t) d t}
$$

respectively. Thus, $C_{v}$ is given by $\sigma_{t} / \bar{t}$. It is known that $C_{v}$ takes values of 0 and 1 for regular and complete random spikes, respectively [34]. Figure 4 shows the value of $C_{v}$ in the same parameter range as Fig. 3. The firing rate of $V_{4}$ becomes zero (Fig. 3) and $C_{v}$ for $V_{1}, V_{2}$, and $V_{5}$ becomes zero (Fig. 4) at the same value of $G_{s y n_{14}} \simeq 2.7$. We can see that when $G_{s y n_{14}}$ is larger than about 2.7, neurons 1, 2, and 5 produce almost periodic spikes.

We show the waveforms of the membrane potentials at $G_{s^{\prime} n_{14}}=3.0$ in Fig. 5. Figure 5(a) represents antiphase synchronization between $V_{1}$ and $V_{5}$. On the other hand, $V_{1}$ (or $V_{5}$ ) and $V_{2}$ are not synchronized (see Fig. 5(b)). Figure 5(c) shows the waveforms of the remaining neurons $\left(V_{3}, V_{4}\right.$, and $\left.V_{6}\right)$, which have an in-phase subthreshold oscillation. Comparing Figs. 5(a), 5(b), and 5(c), we see that Figs. 5(a) and 5(b) are almost periodic waveforms

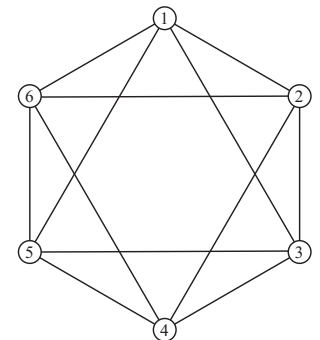

(a) Regular network (second-nearest-neighbor coupling)

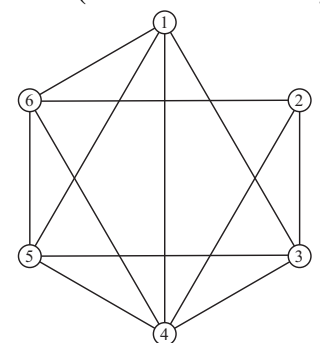

(b) Network obtained by reconnecting one synapse: 1-2 is cut and 1-4 is rewired from (a).

Fig. 1 Networks

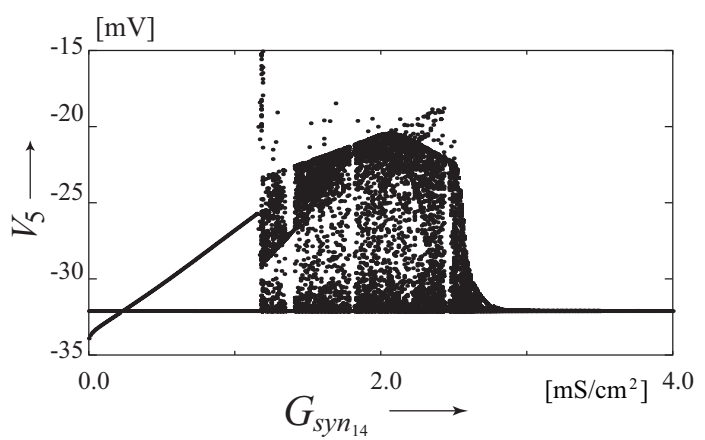

Fig.2 One-parameter bifurcation diagram $\left(I_{e x t}=78.55\right)$

and Fig. 5(c) is non-periodic. Figure 6 shows the result of a fast Fourier transform (FFT) of the membrane potentials. Black, red, and blue curves indicate the FFTs of $V_{1}$, $V_{2}$, and $V_{3}$, respectively. The black and red curves have main peaks $\left(f_{V_{1}}\right.$ and $\left.f_{V_{2}}\right)$ and higher-harmonic components. The blue curve shows that $V_{3}$ has two main frequencies between those of $V_{1}$ and $V_{2}\left(f_{V_{2}}\right.$ and $\left.2 f_{V_{1}}\right)$. From these FFT results, we can see that the membrane potentials of neurons 1 and 2 are almost periodic and that neuron 3 produces a quasi-periodic spike train. In other words, the frequency components of the quasi-periodic solution are decomposed to those of neurons 1 and 2 .

\subsection{BVP model}

Almost the same phenomenon as that described in Sect. 3.1 is observed for the synaptically coupled BVP equations (Eq. (3)). Here, "almost the same" means 


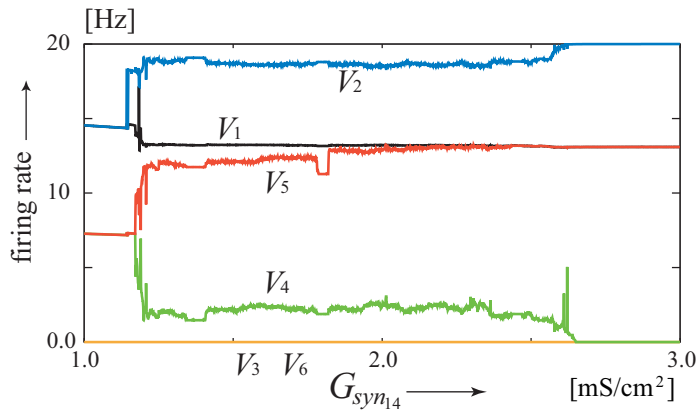

Fig. 3 Average firing rate for 2000 spikes $\left(I_{e x t}=78.55\right)$

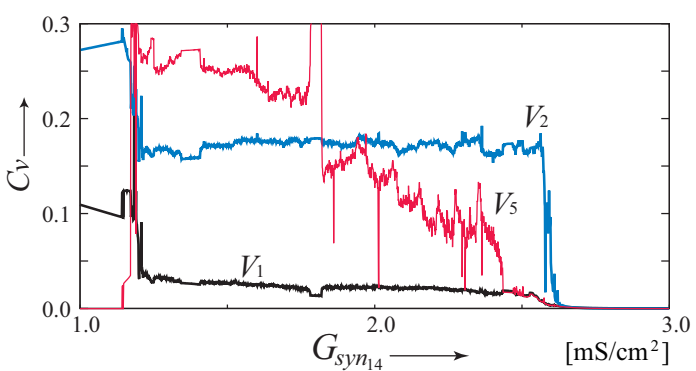

Fig. 4 Coefficient of variation for membrane potentials of $V_{1}, V_{2}$, and $V_{5}\left(I_{e x t}=78.55\right)$

that there are three clustered groups: two groups consisting of fired neurons with distinct firing frequencies and one group with only subthreshold oscillations. The BVP equations are derived from the simplification of the Hodgkin-Huxley equations. Although the dimension of the equations is the same as that of the ML equations, the dynamics is much simpler. Figure 7(a) shows phasesynchronized waveforms of $x_{1}$ and $x_{5}$. On the other hand, $x_{1}$ and $x_{2}$ are not synchronized. The remaining elements have beat oscillations as shown in Fig. 7(c). This waveform shows a typical quasi-periodic solution. Since neurons 1,2 , and 5 are connected through the weak synaptic currents of neurons 3,4 , and 6 , the amplitudes of $x_{1}$ and $x_{5}$ are different. The Fourier spectra (Figs. 8(a) and 8(b)) prove the fact that $x_{1}$ and $x_{2}$ are almost periodic states, and $x_{3}$ is a quasi-periodic state (there are two main frequencies).

\subsection{Large population}

\subsubsection{Identical neurons}

We showed the results for $n=6$ in the previous subsections. In that case, the clustered states are a pair of connected neurons (neurons 1 and 5), one neuron (neuron 2) that is almost isolated from the network, and neurons with only subthreshold oscillations. Here, we show

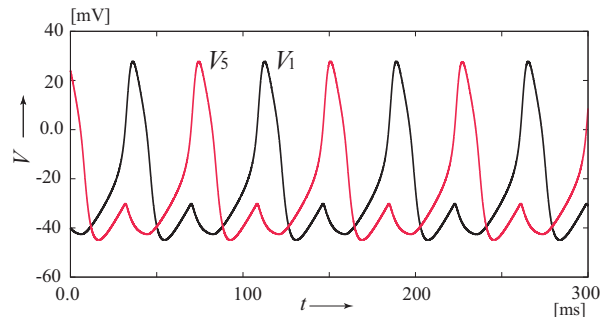

(a) $V_{1}$ (black) and $V_{5}$ (red)

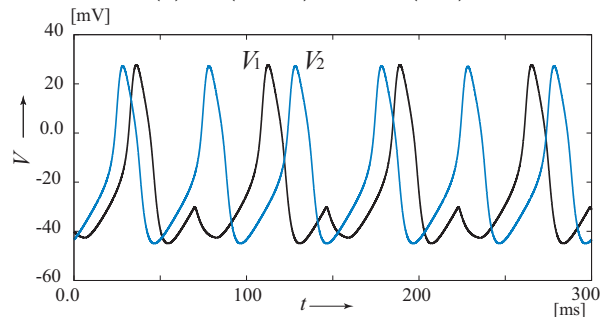

(b) $V_{1}$ (black) and $V_{2}$ (blue)

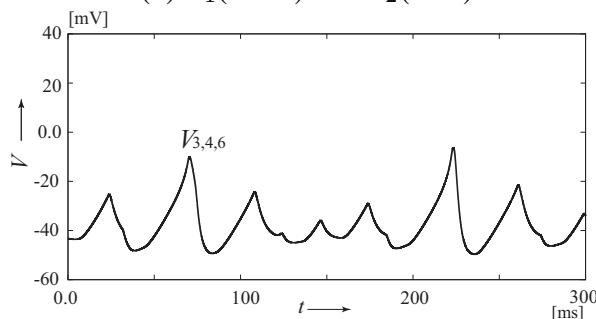

(c) $V_{3}, V_{4}$, and $V_{6}$

Fig. 5 Waveforms of membrane potentials for ML neurons with $G_{\text {syn }_{14}}=3.0$

the results for a larger system. We made a regular onedimensional ring of $n$ neurons (here, $n=24$ ) with connections up to the second-nearest neighbors. Using the random rewiring procedure [1], we constructed a system with the small-world property (high clustering and short path length) from the regular lattice. We set the number of rewired connections to 10 to satisfy the small-world property. We use two kinds of synaptic conductance: $G_{s y n_{1}}$ for rewired connections and $G_{s y n_{2}}$ for others. The resultant network structure is shown in Fig. 9.

Figure 10 shows a one-parameter bifurcation diagram. We change the values of the synaptic conductances $G_{s y n_{1}}$ of the 10 rewired connections and fix the value of $G_{s y n_{2}}$ as 3.75. We observe a similar phenomenon to that for $n=6$ around $G_{s n_{1}}=3.75$. That is, the neurons with the lower firing frequency are suppressed by the neurons with the higher firing frequency.

At $G_{s y n_{1}} \simeq G_{s y n_{2}}$ we obtain three clustered groups as shown in Fig. 9 [28]. In this state, there are two groups that exhibit firing: directly connected blue nodes and indirectly connected red nodes. Figure 11 shows waveforms of the membrane potentials for the two groups. The connected blue pairs show antiphase synchronization (neurons 13 and 17, and neurons 9 and 14 are in-phase) (Fig. 


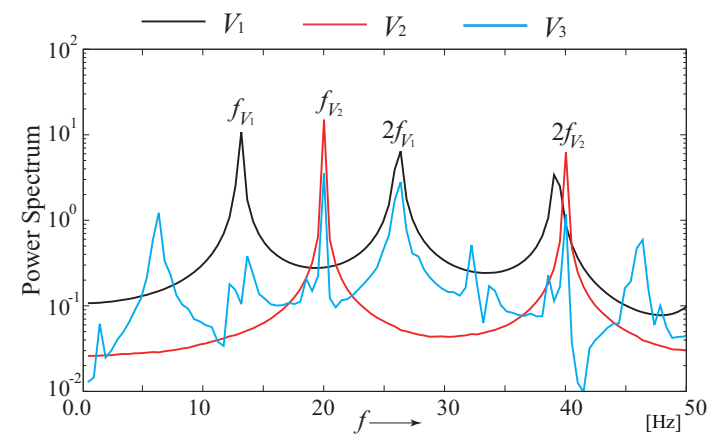

Fig. 6 FFT of membrane potentials for ML neurons

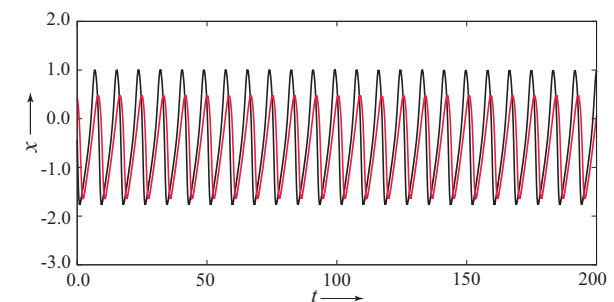

(a) $x_{1}$ (black) and $x_{5}$ (red)

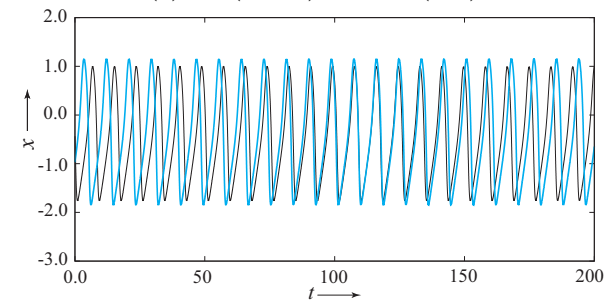

(b) $x_{1}$ (black) and $x_{2}$ (cyan)

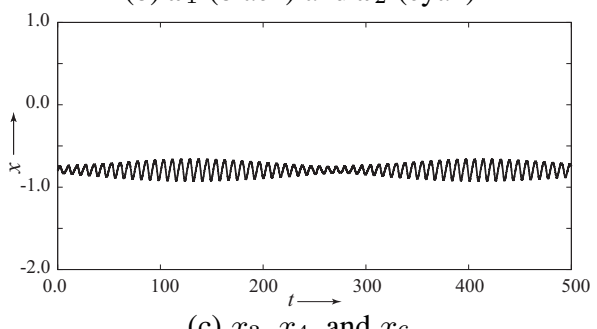

(c) $x_{3}, x_{4}$, and $x_{6}$

Fig. 7 Waveforms of $x_{i}$ for BVP

11(b)) and all the red pairs are phase-synchronized (Fig. 11(a)). Thus, two main firing frequencies exist as in the case of $n=6$ shown in Sect. 3.1.

\subsubsection{Non-identical neurons}

We consider that one of the important factors in generating a firing pattern shown in the previous subsection is the existence of neurons with only subthreshold oscillations. Next, we decrease the value of the DC current for each neuron to 40 (no firing) to obtain a neuron with only subthreshold oscillations. From the results in Sect. 3.1 , we know that neurons with a higher firing frequency

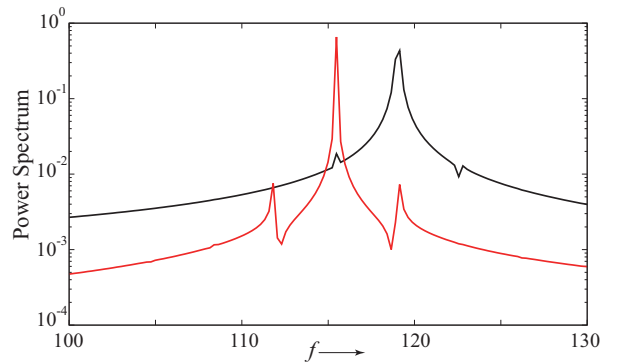

(a) $x_{1}$ (black) and $x_{2}$ (red)

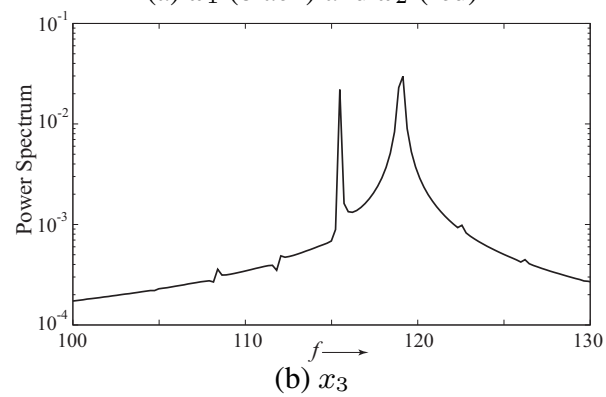

Fig. 8 FFT of $x_{i}$ for BVP

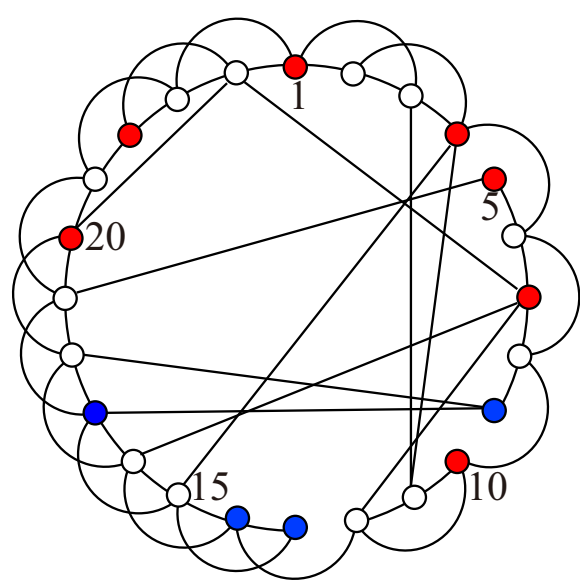

Fig. $9 n=24$ : Red and blue colored nodes have distinct firing frequencies. White nodes have subthreshold oscillation for $I_{\text {ext }}=71.0, G_{s y n_{1}}=G_{s y n_{2}}=3.75$.

suppress neurons with a lower firing frequency. Thus, the neuron with the decreased DC current cannot be forced to fire, then we count the firing patterns of the other neurons. As a result, we observe eight patterns. The decreased DC current of one neuron is a control signal to recall one of the eight firing patterns.

\section{Conclusion}

We investigated the synchronized firings in networks composed of inhibitory ML or BVP neurons. First, we studied the simplest model in which we can reconnect 


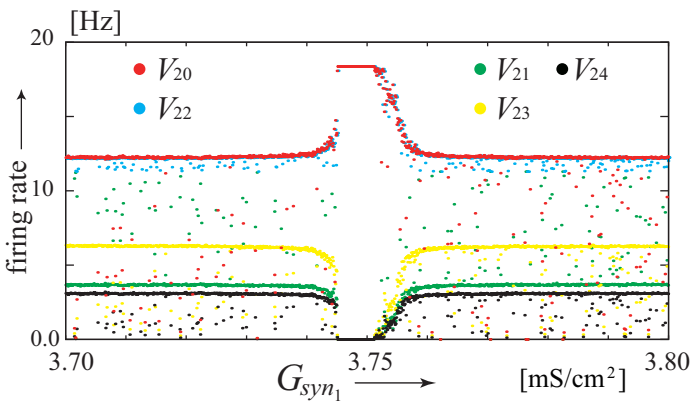

Fig.10 One-parameter bifurcation diagram for $n=24$

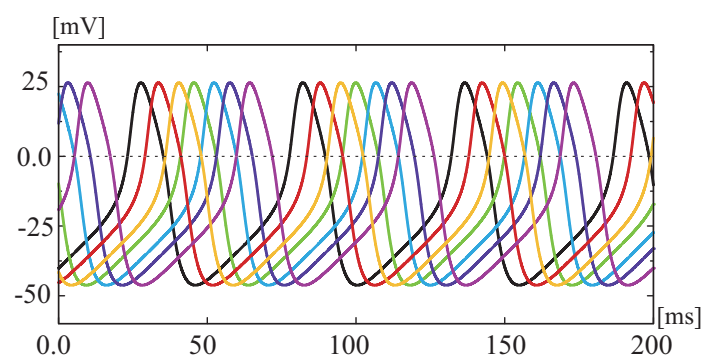

(a) Phase-synchronized states (red neurons in Fig. 9) $[\mathrm{mV}]$

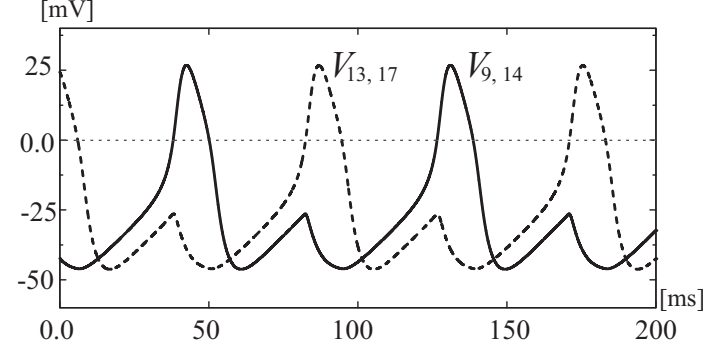

(b) Phase-synchronized states (blue neurons in Fig. 9): A pair of neurons is synchronized at the in-phase

Fig. 11 Waveforms of membrane potentials in Fig. 9

synapses. When we reconnected only one synapse, an interesting quasi-periodic solution was observed. It contains three clustered groups: two groups consisting of neurons with distinct firing frequencies and one group with only subthreshold oscillations. The neurons in the former groups produce almost periodic spikes (the periodicity is judged by the coefficient of variation of interspike intervals and Fourier spectra). However, the neurons in the latter group produce quasi-periodic spikes. In the Fourier spectrum of this solution, there are two main peaks composed of the frequencies of the almost periodic spikes. A similar phenomenon was observed in a large network with the small-world property. From the study of the bifurcation structure, we can see that the mechanism for generating clustered states with quasi-periodicity is as follows: (1) non-uniformity of the firing frequency appears as a result of asymmetrical coupling, (2) neurons with a higher firing frequency suppress neurons with a lower firing frequency for an appropriate coupling coefficient, (3) some neurons only connected to neurons that do not fire are isolated from the network, (4) three clustered states, that is, isolated neurons with firing frequency $\mathrm{A}$, directly connected neurons with frequency $\mathrm{B}(\mathrm{A} / \mathrm{B}$ is irrational), and neurons that do not fire, are generated.

Moreover, we observed many firing patterns in a large population. These firing patterns may correspond to stored memories in the brain. We determined that the control input (the DC current applied to a neuron) can recall the desired patterns. The key to generating such firing patterns is the existence of neurons with only subthreshold oscillations. Subthreshold neuronal oscillations have been observed in previous experiments [35,36]. Our phenomenon shows the importance of the subthreshold oscillation to connect the neurons with periodic spikes. Also, this result indicates that real connections in neuronal networks are more complicated than the estimated connectivity from observed spike trains.

Studying bifurcations of quasi-periodic solutions using the methods in $[37,38]$ is an open problem that we are tackling. Also, we are studying systems with different network structures (different numbers of neurons and reconnecting synapses).

\section{Acknowledgment}

This work was supported by JSPS KAKENHI Grant Number 15K00405.

\section{References}

[1] D. J. Watts and S. H. Strogatz: Collective dynamics of small world networks, Nature, Vol. 393, pp. 440 442, 1998.

[2] A. L. Barabási and R. Albert: Emergence of scaling in random networks, Science, Vol. 286, pp. 509$512,1999$.

[3] R. Albert and A. L. Barabási: Statistical mechanics of complex networks, Rev. Mod. Phys., Vol. 74, pp. 47-97, 2002.

[4] D. S. Bassett, A. M. Lindenberg, S. Achard, T. Duke and E. Bullmore: Adaptive reconfiguration of fractal small-world human brain functional networks, Proc. Natl. Acad. Sci. U.S.A., Vol. 103, pp. 19518-19523, 2006.

[5] L. Zemanová, C. Zhou and J. Kurths: Structural and functional clusters of complex brain networks, Physica D, Vol. 224, pp. 202-212, 2006.

[6] C. M. Gray, P. König, A. K. Engel and W. Singer: Oscillatory responses in cat visual cortex exhibit inter-columnar synchronization which reflects 
global stimulus properties, Nature, Vol. 338, No. 6213, pp. 334-337, 1989.

[7] A. Pikovsky, M. Rosenblum and J. Kurths: Synchronization: A Universal Concept in Nonlinear Sciences, Cambridge University Press, 2001.

[8] M. Barahona and L. M. Pecora: Synchronization in small-world systems, Phys. Rev. Lett., Vol. 89, No. 5, 054191, 2002.

[9] T. Nishikawa and A. E. Motter: Maximum performance at minimum cost in network synchronization, Physica D, Vol. 224, pp. 77-89, 2006.

[10] L. F. Lago-Fernández, R. Huerta, F. Corbacho and J. A. Sigüenza: Fast response and temporal coherent oscillations in small-world networks, Phys. Rev. Lett., Vol. 84, No. 12, pp. 2758-2761, 2000.

[11] H. Hong, M. Y. Choi and B. J. Kim: Synchronization on small-world networks, Phys. Rev. E, Vol. 65, 026139, 2002.

[12] A. M. Batista, S.E.S. Pinto, R. L. Viana and S. R. Lopes: Mode locking in small-world networks of coupled circle maps, Physica A, Vol. 322, pp. 118128, 2003.

[13] H. Hong, B. J. Kim, M. Y. Choi and H. Park: Factors that predict better synchronizability on complex networks, Phys. Rev. E, Vol. 69, 067105, 2004.

[14] T. Nishikawa, A. E. Motter, Y. C. Lai and F. C. Hoppensteadt: Heterogeneity in oscillator network: Are smaller worlds easier to synchronize?, Phys. Rev. Lett., Vol. 91, 014101, 2003.

[15] I. Belykh, E. Lange and M. Hasler: Synchronization of bursting neurons: What matters in the network topology, Phys. Rev. Lett., Vol. 94, 188101, 2005.

[16] M. Zhao, T. Zhou, B. H. Wang, G. Yan, H. J. Yang and W. J. Bai: Relations between average distance, heterogeneity and network synchronizability, Physica A, Vol. 371, pp. 773-780, 2006.

[17] A. Arenas, A. Diaz, J. Kurths, Y. Moreno and C. Zhou: Synchronization in complex networks, Phys. Rep., Vol. 469, pp. 93-153, 2008.

[18] D. Golomb and J. Rinzel: Clustering in globally coupled inhibitory neurons, Physica D, Vol. 72, No. 3, pp. 259-282, 1994.

[19] C. van Vreeswijk: Partial synchronization in populations of pulse-coupled oscillators, Phys. Rev. E, Vol. 54, 5522, 1996.
[20] X. J. Wang and G. Buzsáki: Gamma oscillation by synaptic inhibition in a hippocampal interneuronal network model, J. Neurosci., Vol. 16, No. 20, pp. 6402-6413, 1996.

[21] H. Markram, M. Toledo-Rodriguez, Y. Wang, A. Gupta, G. Silberberg and C. Wu: Interneurons of the neocortical inhibitory system, Nat. Rev. Neurosci., Vol. 5, No. 10, pp. 793-807, 2004.

[22] V. S. Sohal and J. R. Huguenard: Inhibitory coupling specifically generates emergent gamma oscillations in diverse cell types, Proc. Natl. Acad. Sci. U.S.A., Vol. 102, No. 51, pp. 18638-18643, 2005.

[23] S. Boccaletti, V. Latora, Y. Moreno, M. Chavez and D. U. Hwang: Complex networks: Structure and dynamics. Phys. Rep., Vol. 424, No. 4, pp. 175-308, 2006.

[24] B. Ermentrout and M. Wechselberger: Canards, clusters, and synchronization in a weakly coupled interneuron model, SIAM J. Appl. Dyn. Syst, Vol. 8, No. 1, pp. 253-278, 2009.

[25] X. Liang, M. Tang, M. Dhamala and Z. Liu: Phase synchronization of inhibitory bursting neurons induced by distributed time delays in chemical coupling, Phys. Rev. E, Vol. 80, No. 6, 066202, 2009.

[26] A. L. Person and I. M. Raman: Purkinje neuron synchrony elicits time-locked spiking in the cerebellar nuclei, Nature, Vol. 481, No. 7382, pp. 502-505, 2012.

[27] B. Haider, M. Haüsser and M. Carandini: Inhibition dominates sensory responses in the awake cortex, Nature, Vol. 493, No. 7430, pp. 97-100, 2013.

[28] H. Kitajima: Synchronization in synaptically coupled neurons with small-world structure, Proc. IEEE/NDES'09, pp. 70-73, June 2009.

[29] C. Morris and H. Lecar: Voltage oscillations in the barnacle giant muscle fiber, Biophys. J. Vol. 35, pp. 193-213, 1981.

[30] T. Tateno and H.P.C. Robinson: Rate coding and spike-time variability in cortical neurons with two types of threshold dynamics, J. Neurophysiol. Vol. 95, pp. 2650-2663, 2006.

[31] R. Fitzhugh: Impulses and physiological states in theoretical models of nerve membrane, Biophys. J., Vol. 1, No. 6, pp. 445-466, 1961.

[32] H. Kawakami: Dynamics of Biological Rhythmic Phenomena, Corona Pub., 2001 (in Japanese). 
[33] E. Ioka, H. Kitajima and K. Tsumoto: Synchronization of bidirectionally coupled neurons with asymmetrical inputs, J. Signal Process., Vol. 12, No. 4, pp. 287-290, 2008.

[34] W. R. Softky and C. Koch: The highly irregular firing of cortical cells is inconsistent with temporal integration of random EPSPs, J. Neurosci., Vol. 13, No. 1, pp. 334-50, 1993.

[35] Y. Gutfreund, Y. Yarom and I. Segev: Subthreshold oscillations and resonant frequency in guineapig cortical neurons: Physiology and modeling, J. Physiol. Vol. 483, No. 3, pp. 621-640, 1995.

[36] G. Boehmer, W. Greffrath, E. Martin and S. Hermann: Subthreshold oscillation of the membrane potential in magnocellular neurones of the rat supraoptic nucleus, J. Physiol. Vol. 526, No. 1, pp. $115-128,2000$

[37] K. Kamiyama, M. Komuro and T. Endo: Algorithms for obtaining a saddle torus between two attractors, Int. J. Bifurc. Chaos, Vol. 23, No. 9, 1330032, 2013.

[38] K. Kamiyama, M. Komuro, T. Endo and K. Aihara: Classification of bifurcations of quasi-periodic solutions using Lyapunov bundles, Int. J. Bifurc. Chaos, Vol. 24, No. 12, 1430034, 2014.

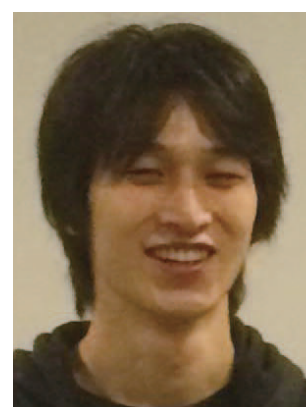

Kaiji Kato received his B.E. degree from the Department of Reliabilitybased Information System Engineering, Faculty of Engineering, Kagawa University, Japan, in 2015. His research interests include bifurcation problems in neuronal systems.

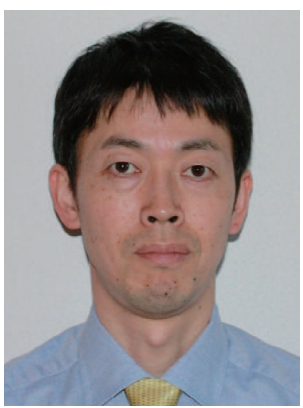

Hiroyuki Kitajima received his B.E., M.E. and Dr.Eng. degrees in electrical and electronic engineering from Tokushima University, in 1993, 1995 and 1998, respectively. $\mathrm{He}$ is a Professor in the Department of Electronics and Information Engineering, Kagawa University. He is interested in bifurcation prob-

lems.

(Received October 13, 2015; revised January 19, 2016) 